\title{
Interception of moving objects in karate: an experimental, marker-free benchmark
}

\section{Giada Romano \\ Davide Viggiano}

Medicine and Health Sciences, University of Molise, Italy

Corresponding author:

Davide Viggiano

Medicine and Health Sciences, University of Molise

Via De Sanctis,

86100 Campobasso, Italy

E-mail: davide.viggiano@unimol.it

\section{Summary}

Background: karate requires an optimal interception of the opponent's attack. Particularly in unconstrained situations, normal, untrained, subjects solve this problem adopting rather different solutions. It is currently unknown if karate as show a more uniform selection of interception schemes due to their special training. Methods: here we applied a 3D scanner to study the movement reproducibility of skilled karate as in a natural setup, using an unconstrained interception task. Six right handed karatekas and six controls participated to the study. 3D motion tracking data of the upper limbs were obtained using the Microsoft Kinect sensor, a real-time 3D scanner. The interception task consisted of intercepting and stopping a moving stick which was directed towards the side of the subject in two different positions (upper and lower). Results: the analysis of hand trajectories showed that the strategy of the movement was remarkably different between control subjects, whereas it was more uniform in karatekas. Moreover, we observed a significant difference in the variability of the interception point between control subjects and karatekas. Conclusion: the results confirm the presence of individual idiosincratic behavior in interception tasks also in ecologically realistic situations, and that experience and training (as in karatekas) play an important role in the trajectory in interceptive tasks.

KEY WORDS: motion tracking, trajectory, eye-hand coordination.

\section{Introduction}

In contact sports such as karate, the interception of moving objects is pivotal in a successful competition. However, the problem of a correct interception of a moving object can lead to an infinite number of solutions, because the interception can occur at any place along the trajectory of the moving object; moreover, one can use many different movements to reach the same final interception point.

Previous work showed that our brain tries to simplify the problem by limiting the possible movements of the arm. For example, shoulder and elbow joints are not really independent in their movements, and the nervous system 'prefers' to move them according to precise relations ${ }^{1-3}$. Moreover, the brain tries to adopt movements that minimize energy, smoothness and accuracy of the movement ${ }^{4}$. Specifically, rather than moving all joints, the brain modifies the position of only few joints, leaving those less relevant for successful performance unchanged ${ }^{5}$.

In general, the nervous system has to decide whether to improve:

(i) the spatial accuracy of the movement (where to intercept the moving object, in a precise place), which requires slower movements, or

(ii) the temporal accuracy (when to intercept the moving object, in a precise instant), by increasing the velocity of the hand 6 .

These observations are relevant in sports sciences, and particularly in sports requiring the interception of moving objects, such as volleyball, basketball, tennis, and martial arts.

Specifically, karate as need to successfully intercept or avoid kicks or punches of the opponents.

In normal subjects it is possible to observe large intersubject variability in the movement of the arm when intercepting an object: this is due not only to noise or level of expertise ${ }^{7}$, but also to different interception strategies and different sensorial sensitivity 8,9 .

In fact, a recent paper showed that, in normal subjects, the spatiotemporal control of a collision is not univocally specified by the brain, so that different subjects adopt different but equally successful solutions ${ }^{8}$. This result is extremely interesting given the necessity for karateka to optimize the trajectories both in time and space, which is expected to result in a lower variability in the movements compared to normal subjects.

Classically this problem has been addressed using motion tracking systems in laboratory situations, which may be very far from the real competitions: mo- 
tion tracking systems usually require the application of reflective markers and to wear specific clothes in a specific environment.

In the present paper we explore the study of this problem in an ecologically valid situation, using a cost-effective 3D scanner which is completely marker-free and can be used in any open space. As other low cost devices ${ }^{10}$, this scanner (the Microsoft Kinect) is an interesting technology which uses patterned light to derive the depth of different segments of the human body without external markers. This device has been previously validated for postural control ${ }^{11}$, for personalization of exercises ${ }^{12}$, foot posture ${ }^{13}$, hand tracking ${ }^{14,15}$.

Here we applied this technology to the study of movement reproducibility of skilled karate as in a natural setup, using an unconstrained catching task. To this aim, we recorded upper limb kinematics using this label-free motion tracking system while the karate as executed a interception task, consisting in the interception of a human moved stick towards a target close to the subject.

The results are of interest given that previous observations on interception tasks were conducted on normal subjects and usually were based on catching a flying ball. Very few studies were conducted in a more ecologically valid environment and none regarded karatekas.

Previous works on karatekas moving in an ecologically valid environment were mostly aimed at identifying kinematic variables characterizing winner karatekas ${ }^{16}$ and no data are currently available on the variability of the execution of the athletic gesture in this sport.

\section{Materials and methods}

\section{Subjects}

Six right handed karatekas (age $25 \pm 10$ ys, weight $61 \pm 8.0 \mathrm{Kg}$, height $160 \pm 2.0$, BMI $23.8 \pm 2.1$ mean \pm SD) and six controls (age $31 \pm 16 \mathrm{ys}$, weight $63 \pm 7.6 \mathrm{Kg}$, height $159 \pm 2 \mathrm{~cm}$, BMI $24.9 \pm 2.4$ ) participated to the study. The karatekas had an equal number of years of regular training experience in karate with regular training 2-3 times a week (second degree black-belt). The participants were healthy with no history of neurological conditions or medication use that would have influenced their postural control volunteered to participate. The study design conformed to the Declaration of Helsinki and was conducted after approval from the local Ethics Committee ${ }^{17}$. The procedures, risks and goals were explained to the participants and their parents, and written consent was obtained prior to commencement of the study.

\section{Motion tracking}

3D motion tracking data were obtained from the Microsoft KinectTM, a real-time 3D scanner, able to record human movements without markers ${ }^{11,13}$.
The Microsoft KinectTM system provides real-time anatomical landmark position data in three dimensions (3D). It is portable and simple to setup, and has good inter-trial reliability in reching tasks, compared to gold standard motion tracking systems such as the Vicon ${ }^{11}$. The Kinect sensor was connected to the USB port of a notebook [Intel(R) Pentium ® CPU B950, 2.10 Ghz, 4GB ram, 64bit operating system] running the Windows7 operating system.

Data from the sensor were collected using Virtual Sensei software (www.virtualsensei.it), based on the OpenNI libraries (http://www.openni.org/). The software was used to acquire the skeleton joint position data in three axes (converted to $\mathrm{X}=$ mediolateral, $\mathrm{Y}=$ anteroposterior, $\mathbf{Z}$ = vertical) which were exported as a comma separated variable (.csv) file. These data were acquired at approximately $20 \mathrm{~Hz}$.

The trials were performed in a position $1.15 \mathrm{~m}$ in front of the Microsoft KinectTM, which was placed on a tripod at a height of $1 \mathrm{~m}$ from the floor (Fig. 1). At this distance the legs were outside the field of view of the sensor, but the precision and quality of the movement detection of the arms was better.

The sensor was calibrated by recording the skeleton joint position of a subject at the same distance of the subjects under investigation (see below) and measuring the distance between the hands as a reference known distance.

\section{Procedure}

To allow the karatekas to perform an ecologically valid environment, they were asked to wear their tracksuit.

The procedure was organized in order to reproduce a real situation (defensive movements) in an uncon-

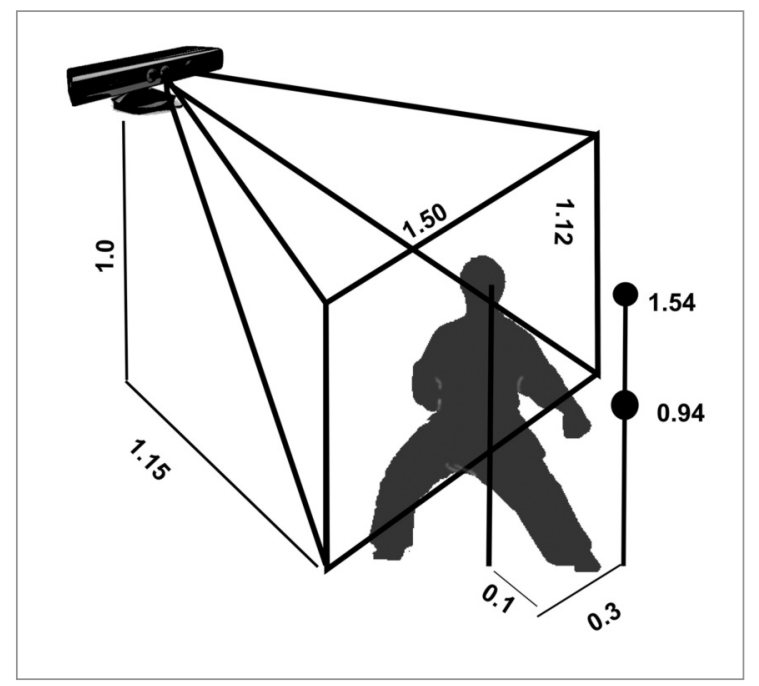

Figure 1. Experimental apparatus. Subjects were standing at a distance of $1.15 \mathrm{~m}$ in front of the Kinect sensor with two white markers positioned behind the subject, indicating to an attacker the possible targets of the attack. 
strained task, with some compromise between the reproducibility of the experiment and an ecologically valid hitting movement.

The task consisted in intercepting and stopping a moving stick which was directed towards the side of the subject in two different positions (upper and lower).

We will refer to the experimenter required to move the stick towards the karateka as the attacker and to the karateka (and the control subjects) as the defender.

Each subject performed two interception tasks (upper and lower reach) in a random order.

To perform the defensive action, the defender was required to:

(1) start in a slight modification of the position "zenkutsu dachi", with the left leg bent and in front, the right leg straighten and in the back and the arms beside the body. The position of the feet and the distance between them $(45 \mathrm{~cm})$ was kept constant for all subjects using two markers applied on the floor. The feet were lined at $45^{\circ}$ from the axis of the kinect sensor (Fig. 1). They were instructed to be prepared to intercept a stick moving towards their right side

(2) reach the stick with the right hand while keeping both feet on the floor, and

(3) return to the starting position.

All tests were performed a minimum of twenty times. The subjects were accostumed with the task during at least ten trials before the recording session begun.

The attacker was instructed to move a $1 \mathrm{mt}$ long rigid stick towards two possible round targets (white, 1.6 $\mathrm{cm}$ diameter) located behind the karateka. Considering the subject's trunk as a reference, the targets were located $10 \mathrm{~cm}$ behind the subject and $30 \mathrm{~cm}$ on the right; the upper target was located at the height of $1.54 \mathrm{~m}$, approximately at the level of the defendor's shoulder; the lower target was located at the height of $0.94 \mathrm{~m}$, approximately at the level of the defensor's hip. Since the targets were behind the defender, the karatekas could not see their location during the task. To improve the reproducibility of the movement of the attacker, we decided to maintain the height of the two white targets fixed for all defenders. However, since this could have introduced differences in the responses of the subjects simply due to their different height, we selected subjects in a very narrow range of heights, in order to obtain roughly the same position of the markers compared to the defenders' body.

The attacker was standing on one side of the karate$\mathrm{ka}$, at a distance of $1.5 \mathrm{~m}$, outside the field of view of the kinect sensor.

We were forced to use a stick as hitting system and not the very same hands of the attacker (which would result in a even more natural setup) due to a limitation of the Kinect sensor: if the hands of the attacker arrive close to the defender, these are not well resolved by the sensor (due to occlusion or mixing) and therefore the quality of the tracking was drastically reduced. Vice versa, using a small stick there was no interference in the tracking process.

The same attacker was used for all subjects in this experiment.
The attacker was wearing headphones connected to a MP3 reader which conveyed a random sequence of two possible sounds with different pitch.

The attacker was then instructed to:

(1) start in a position with the arms beside their body and the stick vertically placed, and to be prepared to move the stick once the sound appeared. He was keeping the feet immobile and was required to fixate at a predetermined location behind the defender, and never looking at the hitting targets throughout the experiment;

(2) once a sound signal appeared (which could be listened only by the attacker) he immediately had to start the movement of the stick either towards the upper target (high pitch sound) or lower target (low pitch sound). The sounds appeared at random time intervals ranging from 1 to 10 seconds and the sequence high-pitch low-pitch was also random so that the defender could not predict when and where the stick was going to move. The velocity of the stick was unconstrained and could change from trial to trial;

(3) return to the starting position once the stick touched the target or the defender intercepted the stick.

In order to allow an ecological situation, the karateka could look at the attacker, whereas the attacker was required to fix behind the karateka and never to watch the targets (which could predict the direction of the movement).

The attacker was previously trained to perform the movement and reached a success rate of $99 \%$.

\section{Data analysis}

A custom program was written in $\mathrm{R}$ environment and used to read in and display the Kinect tracking data.

The interception point was calculated for each trial and for each subject for further analysis. To analyze the variability in the interception point, the distance of each interception point from the mean of all terception points of the same subject was calculated. These distances were then pooled and used to analyze the differences between the karatekas and the control subjects with a t-test for non paired data. Rejection threshold for null hypothesis was set at $p<0.05$.

\section{Results}

Due to the simplicity of the task all subjects were able to intercept the moving stick with comparable performance both when the stick was moved towards the upper or the lower part of the defender.

However, as shown in Figure 2, the strategy of the movement was remarkably different between control subjects, whereas it was more comparable in karatekas. Specifically, it is evident that the hand trajectory was not a straight line, but followed a curved or even a sinusoidal pattern in control subjects whereas it was more linear in karatekas and usually occurred along a defined trajectory. 


\section{Ctl}
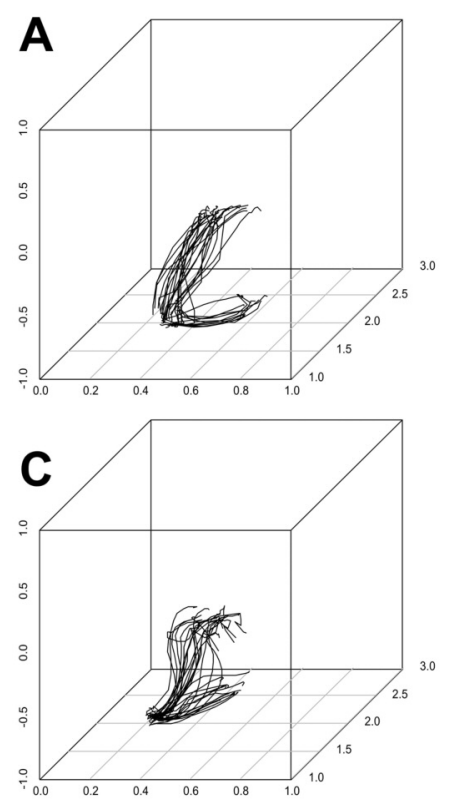

\section{Karateka}
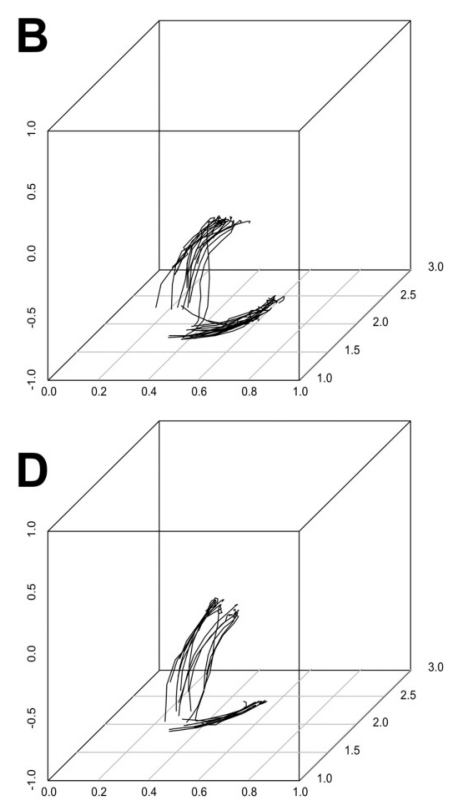

Figure 2. Example of hand trajectories in two control subjects and two karatekas. The movements to intercept the upper and lower attacks are represented in the same graph. Each line represents a different trial.
To quantitatively analyze the variability of the movement, we then considered the final interception position of the hand in the two groups of subjects. To this aim, we calculated the distance of the interception point from the average of all interception points in the same subject. In other terms, the mean of these distances equals the radius of the sphere where the interception occurred; the larger the sphere, the higher variability of the catching point.

An indipendent sample t-test showed significant differences in the variability of the interception point between control subjects and karatekas when the target of the attacker was the upper region of the defender: the karatekas showed much lower variation in the interception point $(5.1 \pm 0.41 \mathrm{~cm}$ mean \pm standard error $)$ compared to the controls $(9.5 \pm 0.5 \mathrm{~cm}$, mean \pm standard error; $p<0.01$ two-tailed t-test).

At variance, the variability of the interception point, when the target of the attacker was the lower region of the defender, was not significantly different between the two groups (karatekas: $7.2 \pm 0.5 \mathrm{~cm}$; controls: $8.2 \pm 0.6 \mathrm{~cm} ; \mathrm{p}<0.19$ two-tailed t-test).

\section{Discussion}

The main finding of the present study was that the strategy of the movement was remarkably different between control subjects, whereas it was more uniform in karatekas. Moreover, we observed a significant difference in the variability of the interception point between control subjects and karatekas. The results confirm the presence of individual idiosincratic behavior in interception tasks also in ecologically realistic situations, and that experience and training (as in karatekas) play an important role in the final trajectory in interceptive tasks.

This result may be explained taking into consideration that the brain of karatekas responds to sensory stimuli very differently from normal subjects: their eventrelated desynchronization, the low-alpha EEG rythm and the motion-related cortical potentials after spontaneous motion are all significantly different from control subjects as reviewed in excellent articles ${ }^{18}$.

Strengths and weaknesses of the study. The main strength of the study is the use of marker-free motion-tracking technologies, together with the use of ecologically valid procedures. The principal weakness of the study is represented by the need to use a stick to hit the karatekas in the interception task, which may not be completely comparable to a real attack. Strengths and weaknesses in relation to other studies. The study replicates the main results of a previous experiment ${ }^{8}$, that is the great difference in the trajectories between control subjects during an interception task. However this previous observation did not take into consideration the training of the subjects and were limited to the analysis of catching a flying ball. Our observations confirm the previous observations in a different, more ecologically valid, procedure and add information in the case of skilled karatekas. Meaning of the study. We hypothesize that the lower variability observed in skilled karatekas derives from the uniform training of these athletes, so that they use a main motor solution to the interception problem. This result is important for the evaluation of the 
training status of karatekas, as a well trained karateka is also expected to show a low level of variability. Unanswered questions and future research. Many aspects still await to be clarified and could not be addressed in the present work. Specifically, it would be interesting to verify the variability of interception movements directly during competitions and to verify that a higher variability predicts lower performances during competitions.

\section{References}

1. Lacquaniti F, Soechting JF, Terzuolo SA. Path constraints on point-to-point arm movements in three-dimensional space. Neuroscience. 1986;17(2):313-324.

2. Flash T, Hogan N. The coordination of arm movements: an experimentally confirmed mathematical model. J Neurosci. 1985;5(7):1688-1703.

3. Morasso P. Spatial control of arm movements. Exp. brain Res. 1981;42(2):223-227.

4. Berret B, Chiovetto E, Nori F, Pozzo T. Manifold reaching paradigm: how do we handle target redundancy? J Neurophysiol. 2011;106(4):2086-2102

5. Todorov E. Optimality principles in sensorimotor control. Nat Neurosci. 2004;7(9):907-915.

6. Tresilian JR, Plooy AM, Marinovic W. Manual interception of moving targets in two dimensions: performance and spacetime accuracy. Brain Res. 2009;1250:202-217.

7. Yarrow K, Brown P, Krakauer JW. Inside the brain of an elite athlete: the neural processes that support high achievement in sports. 2009;10.

8. Cesqui B, d'Avella A, Portone A, Lacquaniti F. Catching a ball at the right time and place: individual factors matter. PLoS One. 2012;7(2):e31770

9. Osborne LC, Lisberger SG, Bialek W. A sensory source for motor variation. Nature. 2005;437(7057):412-416.

10. D'Addona V, Evangelista M, Viggiano D. A new method for quantitative tremor assessment in sports. Sport Orthop Traumatol. unknown; 2014;30:54-59.

11 Clark RA, Pua Y, Fortin K, Ritchie C, Welster KE, Denehy L, et al. Validity of the Microsoft Kinect for assessment of postural control. Gait Posture. Elsevier B.V.; 2012;36(3):372-377.

12. Jun S, Kumar S, Zhou X, Ramsey DK, Krovi VN. Automation for individualization of Kinect-based quantitative progressive exercise regimen. 2013 IEEE Int. Conf. Autom. Sci. Eng. leee; 2013;243-248.

13. Mentiplay BF, Clark RA, Mullins A, Bryant AL, Bartold S, Paterson K. Reliability and validity of the Microsoft Kinect for evaluating static foot posture. J Foot Ankle Res. Journal of Foot and Ankle Research. 2013;6(1):1.

14. Park S, Yu S, Kim J, Kim S, Lee S. 3D hand tracking using Kalman filter in depth space. EURASIP J. Adv. Signal Process. Springer Open Ltd. 2012;2012(1):36.

15. Weber I, Koch J, Meskemper J, Friedl K, Heinrich K, Hartmann $\mathrm{U}$. Is the MS Kinect suitable for motion analysis? Biomed Tech. (Berl). 2012;57:4452.

16. Chaabène $H$, Franchini $E$, Miarka $B$, Selmi MA, Mkaouer $B$, Chamari $\mathrm{K}$. Time-motion analysis and physiological responses to karate official combat sessions: is there a difference between winners and defeated karatekas? Int J Sports Physiol Perform. 2014;9(2):302-308.

17. Padulo J, Oliva F, Frizziero A, Maffulli N. Muscles, Ligaments and Tendons Journal. Basic principles and recommendations in clinical and field science research. MLTJ. 2013;4:250-252.

18. Nakata H, Yoshie M, Miura A, Kudo K. Characteristics of the athletes' brain: evidence from neurophysiology and neuroimaging. Brain Res. Rev. Elsevier B.V.; 2010;62(2):197-211. 